\title{
Potential of pepper plant accessions for ornamental purposes using diallel analysis
}

\author{
ANGELA M.S. PESSOA ${ }^{1}$, ELIZANILDA R. DO RÊGO ${ }^{1}$, CRISTINE A.P. DOS SANTOS ${ }^{1}$, MICHELLE \\ G. DE CARVALHO ${ }^{1}$, JÚLIO C.P. DE MESQUITA ${ }^{2}$ and MAILSON M. DO RÊGO ${ }^{1}$
}

${ }^{1}$ Universidade Federal da Paraíba, Laboratório de Biotecnologia Vegetal, Rodovia PB-079, 58397-000 Areia, PB, Brazil

${ }^{2}$ Instituto Agronômico de Pernambuco/IPA, Av. General San Martin, 1371, Bongi, 50761-000 Recife, PE, Brazil

Manuscript received on April 19, 2018; accepted for publication on February 26, 2019

How to cite: PESSOA AMS, RÊGO ER, SANTOS CAP, CARVALHO MG, MESQUITA JCP AND RÊGO MM. 2019. Potential of pepper plant accessions for ornamental purposes using diallel analysis. An Acad Bras Cienc 91: e20180379. DOI 10.1590/0001-3765201920180379.

\begin{abstract}
The genus Capsicum is cultivated in various parts of the world, with C. annuum showing high diversity and potential for ornamental purposes and genetic improvement. The aim of this work was to evaluate the combining ability and heterosis of seedling and plant traits in accessions of ornamental pepper and their hybrids. Seven accessions of $C$. annuum were handcrossed and twelve quantitative plant traits were evaluated. The data were subjected to analysis of variance, the Scott-Knott test and diallel analysis using Griffing's method I (fixed model). Heterosis and its components were also determined. Non-additive effects were found for seedling height, cotyledonous leaf length and width; stem diameter and chlorophyll b content. All other traits showed predominance of additive effects. Heterosis effects were found for some hybrids and UFPB134 x UFPB137, UFPB134 x UFPB390, UFPB134 x UFPB004 and UFPB099 x UFPB004 showed seedlings that are more vigorous.
\end{abstract}

Key words: Capsicum annuum, heterosis, general combining ability, specific combining ability.

\section{INTRODUCTION}

Capsicum genus belongs to Solanaceae family and there are more than 30 species in this genus. Capsicum annuum L., C. chinense Jacq., $C$. frutescens L., C. baccatum L. and C. pubescens Ruiz \& Pav. are the cultivated species (Stommel and Bosland 2006). The C. annuum species is the most widely commercialized species worldwide (Rêgo and Rêgo 2016). The phenotypic diversity found in Capsicum species for plant size, leaf and fruit traits can potentially be used in breeding programs for producing fresh fruit flavorings and colorings

Correspondence to: Elizanilda Ramalho do Rêgo

E-mail: elizanilda@cca.ufpb.br

ORCid: http:/orcid.org/0000-0001-7376-7569 for food industry (Stommel 2008, Mongkolporn and Taylor 2011). This great variability enable peppers to be used as potted, bedding and garden plants and cut stems (Stommel and Bosland 2006, Nascimento et al. 2014, Pessoa et al. 2018, Rêgo et al. 2015, Rêgo and Rêgo 2018).

The ornamental use of pepper plants increased in the last years and for this particular use, plants need to be short and have a small canopy (Silva Neto et al. 2014, Pessoa et al. 2018, Rêgo and Rêgo 2016, 2018). Other characteristics such as fast-growth and increased postharvest life should be selected in breeding programs for ornamental purpose (Rêgo et al. 2010, 2015, Segatto et al. 2013, Santos et al. 2013, Nascimento et al. 2015, Rêgo 
and Rêgo, 2016, 2018). To increase the frequency of favorable alleles into a single genotype is the greatest challenge in a breeding program.

Several methods were proposed to predict the performance of progenies in early and advanced generations (Benin et al. 2009). The diallel analysis is one of them and allows for parents selection, and makes it possible to understand the genetic effects in traits determination (Gonçalves et al. 2015). The most used diallel analysis methods are those proposed by Griffing (1956) and Gardner and Eberhart (1966). The Griffing method estimates the effects of general combining ability (GCA), which depends on the concentration of predominantly additive genes, and specific combining ability (SCA), which is dependent on the concentration of non-additive genes (Sprague and Tatum 1942, Benin et al. 2009, Baldissera et al. 2012). The diallel method of Gardner and Eberhart (1966) supplies detailed information about the potential of parents per se and the heterosis manifested in their hybrids, and it is the most widely used method by plant breeders (Ledo et al. 2003, Nascimento et al. 2014).

The aim of the present study was to evaluate the combining ability and heterosis of seedling and plant traits in accessions of potted ornamental pepper (C. annuum L.), with the goal of identifying the best hybrids and parents to obtain new populations.

\section{MATERIALS AND METHODS}

\section{EXPERIMENTAL SITE AND PLANT MATERIAL}

The experiment was performed in a greenhouse in the Plant Biotechnology sector of the Centro de Ciências Agrárias, Universidade Federal da Paraíba (UFPB), Paraíba State, Brazil (Altitude $618 \mathrm{~m}$, latitude $06^{\circ} 57^{\prime} 48^{\prime \prime} \mathrm{S}$ and longitude $35^{\circ} 41^{\prime}$ 30 ” W).

Seven accessions of ornamental chili pepper (C. annuum L.) were used, belonging to the
Germplasm Bank of the CCA-UFPB: UFPB001, UFPB004, UFPB77.3, UFPB099, UFPB134, UFPB137 and UFPB390 (Figure 1), in full diallel cross, selected based on their genetic diversity for fruit and plant traits (Pessoa et al. 2018).

Hand crosses were performed at the preanthesis stage (Do Rêgo et al. 2012). The floral buds were emasculated in the morning, immediately pollinated by placing the pollen from a donor plant on the stigma of an acceptor flower from another plant, identified and covered with aluminum foil to avoid contamination (Nascimento et al. 2012). Fruits were collected when ripe, and seeds were removed.

Seeds from the seven parents and 42 hybrids were sown in polystyrene trays with 128 cells, which were filled with commercial substrate $\left(\right.$ Plantmax $\left.^{\circledR}\right)$. Thirty days after germination, seedlings were transferred to plastic pots containing $900 \mathrm{~mL}$ of the same substrate.

Morpho-agronomic characterization was performed based on the Capsicum descriptors defined by IPGRI (1995). The following traits were evaluated: four seedling traits - seedling height (SH), hypocotyl diameter (HD), cotyledonous leaf length (CL), cotyledonous leaf width (CW); and six plant traits - plant height $(\mathrm{PH})$, stem diameter (SD), first bifurcation height (HFB), canopy width (CAW), leaf length (LL) and leaf width (LW); and two physiological traits - chlorophyll a (CLA) and chlorophyll b (CLB) contents. The tools used to collect data were a digital caliper (Leetools ${ }^{\circledR}$ digital caliper) for measuring seedling and plant traits and a digital chlorophyllometer (ClorofiLOG FALKER $^{\circledR}$ ) for leaf chlorophyll content. Seedling characterization was performed when they were transplanted to the final site, after 30 days of germination. 

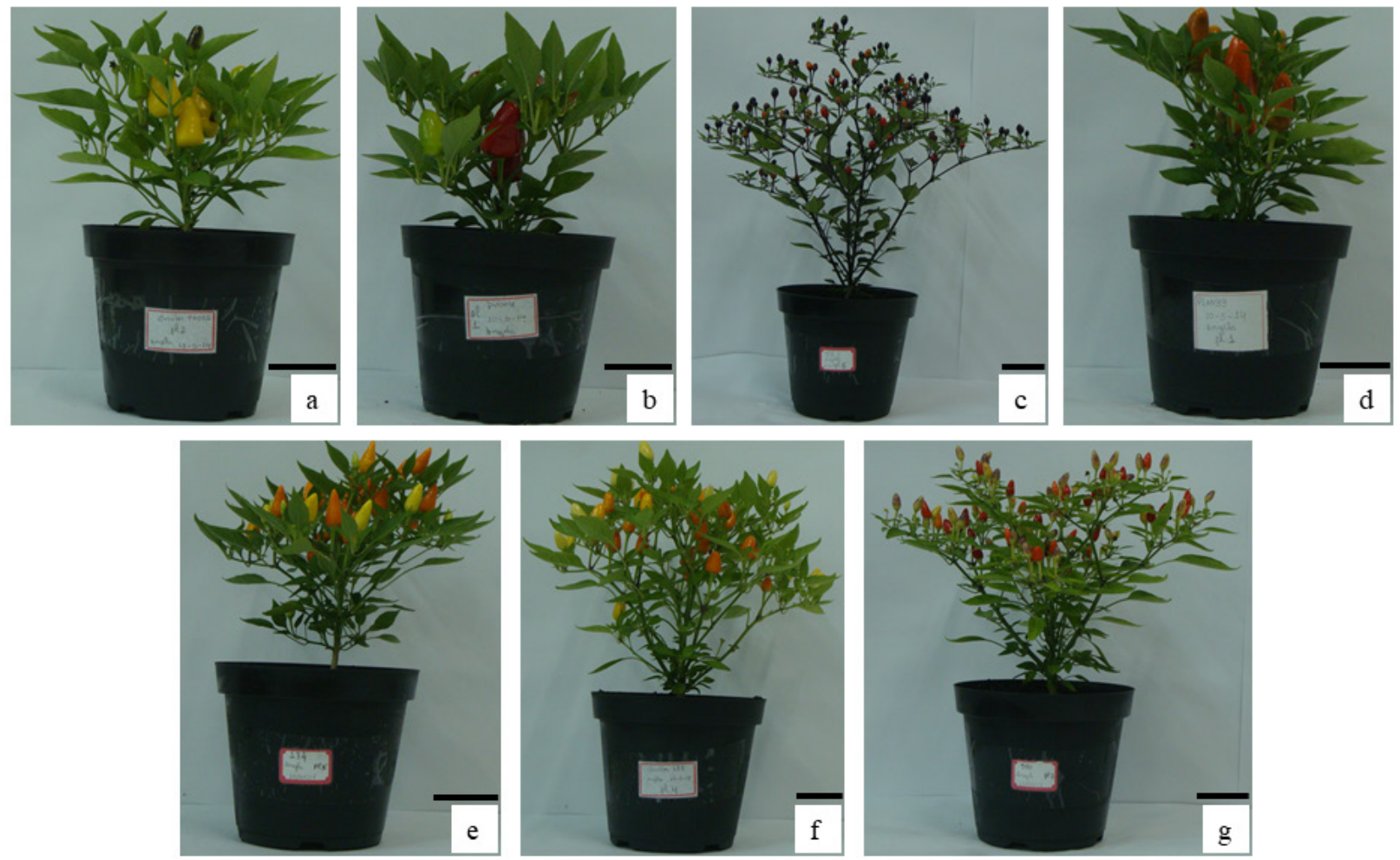

Figure 1 - Genotypes from Capsicum annuum L. a - UFPB001; b - UFPB004; c - UFPB77.3; d - UFPB099; e - UFPB134; f UFPB137 and $\mathbf{g}-$ UFPB390. Bar $=5.0 \mathrm{~cm}$.

\section{STATISTICAL ANALYSIS}

A completely randomized design was used, with 49 treatments (parents and hybrids), and eight replicates per treatment. The data were subjected to analysis of variance, followed by grouping of the means by the Scott-Knott test $(\mathrm{p} \leq 0.01$ or $(\mathrm{p} \leq 0.05)$. All statistical analyses were performed using the software Genes (Cruz 2006).

\section{GRIFFING}

General combining ability (GCA) and specific combining ability (SCA) were estimated using the Griffing's (1956) method I (fixed model). The statistical model was:

$\mathrm{X}_{\mathrm{ijk}}=\mu+\mathrm{g}_{\mathrm{i}}+\mathrm{g}_{\mathrm{j}}+\mathrm{s}_{\mathrm{ij}}+\mathrm{r}_{\mathrm{ij}}+\mathrm{b}_{\mathrm{k}}+\mathrm{e}_{\mathrm{ijk}}$

where: $X_{\mathrm{ijk}}=$ observation value for a cross between the ith and jth parents in the kth replication; $\mu=$ population mean; $g_{i}$ and $g_{j}=$ GCA effect for the ith and jth parents, respectively; $s_{i j}=$ SCA effect for the hybrid between the ith and $j$ th parents; $r_{i j}$ $=$ reciprocal effect for the hybrid; $b \mathrm{k}=$ repetition effect and $\mathrm{e}_{\mathrm{ijk}}=$ experimental error. Significant differences between GCA, SCA and reciprocal effects were tested using the $\mathrm{F}$ test.

\section{GARDNER AND EBERHART}

Heterosis analysis was performed using the Gardner and Eberhart (1966) method. The variety effect (v) was also estimated for each parent using this method. Variety effects and heterosis components, for generation $\mathrm{k}$, were estimated using the following statistical model:

$\mathrm{Y}_{\mathrm{ij}}=\mu+\left(\mathrm{v}_{\mathrm{i}}+\mathrm{v}_{\mathrm{j}}\right) / 2+\theta\left(\mathrm{h}+\mathrm{h}_{\mathrm{i}}+\mathrm{h}_{\mathrm{j}}+\mathrm{s}_{\mathrm{ij}}\right)+\overline{\mathrm{e}}_{\mathrm{ij}}$

where: $\mathrm{Y}_{\mathrm{ij}}=$ observed mean value of the parent $(\mathrm{i}=\mathrm{j})$ or hybrid combination $(i \neq j) ; \mu=$ mean of varieties; $\mathrm{v}_{\mathrm{i}}=$ effect of $\mathrm{i}$ variety; $\mathrm{v}_{\mathrm{j}}=$ effect of $\mathrm{j}$ variety; $\mathrm{h}=$ mean heterosis effect; $h_{i}=$ effect of $i$ variety 
heterosis; $h_{j}=$ effect of $\mathrm{j}$ variety heterosis; $\mathrm{s}_{\mathrm{ij}}=$ effect of specific heterosis; $\overline{\mathrm{e}}_{\mathrm{ij}}=$ mean experimental error; and $\theta=0$, when $\mathrm{i}=\mathrm{j}$, and $\theta=1$, when $\mathrm{i} \neq \mathrm{j}$.

The heterosis estimate was calculated in relation to the parents' means and heterobeltiosis, which is the heterosis in relation to the parent with best average performance, was calculated as well. The following equations 3 and 4 were used:

$$
\begin{aligned}
& H(\%)=\frac{F_{1}-P M}{P M} \times 100 \\
& H(\%)=\frac{F_{1}-S P}{S P} \times 100
\end{aligned}
$$

Where $\mathrm{H}$ is heterosis; $\mathrm{F}_{1}$ is hybrid mean; $\mathrm{PM}$ is parents mean; $\mathrm{Hb}$ heterobeltiosis and $\mathrm{SP}$ is superior parent mean.

\section{RESULTS}

There were significant differences among treatments for all evaluated traits $(p \leq 0.01)$, except for stem diameter. The coefficient of variation $(\mathrm{CV})$ varied from $7.7 \%$ for canopy width to $44.0 \%$ for cotyledonous leaf length (Table I). According to the Scott-Knott test, seedling height presented the highest variability (seven groups); followed by the first bifurcation height (six groups), cotyledonous leaf width, canopy width and plant height (five groups), hypocotyl diameter and leaf width (four groups) and all the other traits formed two groups.

Parent UFPB134 and hybrids UFPB004 $\mathrm{x}$ UFPB134, UFPB004 x UFPB390 and UFPB004 $\mathrm{x}$ UFPB001 presented the highest seedling height, hypocotyl diameter and cotyledonous leaf length. On the other hand, UFPB004 x UFPB099, UFPB77.3 x UFPB137 and UFPB77.3 x UFPB099 presented the highest cotyledonous leaf width (Table SII - Supplementary Material). Regarding plant traits, the smallest values for plant height, first bifurcation height, and canopy width were found in genotypes UFPB001 and UFPB099 and the hybrid $001 \times 099$. For leaf length and leaf width the lowest values were found in genotypes 77.3, 137, 134 and $77.3 \times 137$. The highest chlorophyll a content was found in genitors 77.3 and 137 and in the hybrids $77.3 \times 134$ and $137 \times 134$ and their reciprocal (Table SII).

\section{GRIFFING}

The general combining ability (GCA) effects were significant for all seedling and plant traits, except for stem diameter, according to the $\mathrm{F}$ test $(p<0.01)$ (Table I). The specific combining ability (SCA) effects were significant for seedling height, hypocotyl diameter, cotyledonous width, plant height, first bifurcation height, canopy width and chlorophyll $\mathrm{b}$ content $(\mathrm{p}<0.01$ and $\mathrm{p}<0.05)$. On the other hand, SCA was not significant for cotyledonous leaf length, stem diameter and leaf length. The reciprocal effect was significant for all traits except for cotyledonous leaf length (Table I). There was predominance of non-additive effects for seedling height, cotyledonous leaf length, cotyledonous leaf width; stem diameter and chlorophyll b content. All other traits showed predominance of additive effects (Table I).

The selection based on GCA effects $\left(\hat{g}_{\mathrm{i}}\right)$ for plant traits confirmed the results found in means grouping for all evaluated traits (Table III). Regarding the SCA effects, the indicated hybrids to select dwarf plants are: UFPB390 x UFPB001, UFPB004 x UFPB001, UFPB004 x UFPB099 and UFPB004 x UFPB77.3 (Table III). For leaf length and leaf width, the lowest values were found in genotypes UFPB77.3, UFPB137, UFPB134 and UFPB77.3 x 137. The highest gi value for chlorophyll $\mathrm{a}$ and $\mathrm{b}$ contents were found in genitors 134 and in the hybrids (Sij) UFPB134 x UFPB390, UFPB001 x UFPB390, UFPB004 x UFPB390 (Table SII). 


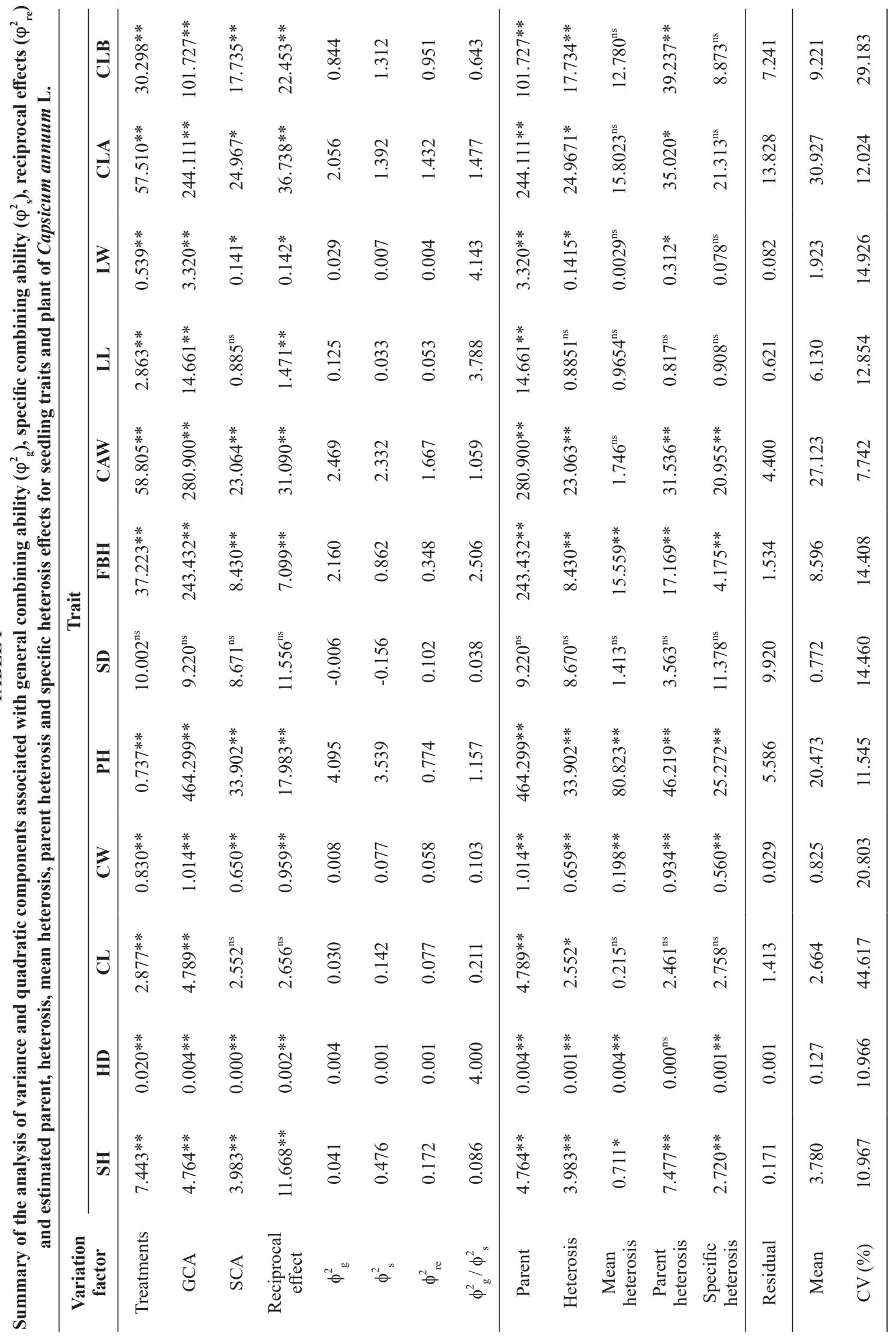




\section{GARDNER AND EBERHART}

Specific heterosis was not significant for cotyledonous leaf length, stem diameter, leaf length, leaf width, chlorophyll a and b contents (Table I). The hybrid UFPB099 $x$ UFPB77.3 presented the highest positive heterosis (82.79) and heterobeltiosis (80.86) for seedling height. The hybrids UFPB390 x UFPB77.3 (14.29) and UFPB004 x UFPB390 (10.88) had the highest positive heterosis, while UFPB77.3 x UFPB099 had the highest positive heterobeltiosis for hypocotyl diameter (13.04) and UFPB004 x UFPB099 had the highest positive heterosis (311.58) and heterobeltiosis (260.00) for cotyledonous leaf width (Table IV). The highest negative heterosis for plant height was observed in UFPB001 x UFPB77.3 (-25.21) and the most negative heterobeltiosis $(-8.95)$ in UFPB001 $\mathrm{x}$ UFPB137 (Table IV). The hybrid UFPB001 x UFPB137 showed the most negative heterosis $(-32.04)$ for the first bifurcation height and the highest negative heterobeltiosis for this trait was observed in UFPB099 x UFPB134 (-34.41) (Table IV). Hybrid UFPB77.3 x UFPB134 presented the most negative heterosis and heterobeltiosis for leaf length (-15.75 and -18.81) and width (-16.32 and -9.12) (Table IV). Hybrid UFPB390 x UFPB137 presented positive heterosis (19.56) and heterobeltiosis (13.40) for chlorophyll a content, while UFPB390 x UFPB001 had the highest positive heterosis (57.21) and heterobeltiosis (51.11) for chlorophyll b content (Table IV).

\section{DISCUSSION}

\section{ANALYSIS OF VARIANCE AND MEAN GROUPING}

The numbers of groups formed for seedling and plant traits indicated variability among the parents and hybrids and that it is possible to obtain gains in a breeding program selecting genotypes in this population. Similar results were found by several authors (Rêgo et al. 2013, Nascimento et al. 2013,
Silva Neto et al. 2014, Pessoa et al. 2015, 2018, Rêgo et al. 2015, Costa et al. 2016). Based on seedling traits, the hybrids UFPB134 x UFPB390, UFPB004 x UFPB390 and UFPB004 x UFPB001 should be selected for seedlings that are more vigorous since their morphometric traits are related to seedling quality (Barroso et al. 2015, 2017, Medeiros et al. 2015, Pessoa et al. 2015). The genotypes UFPB001, UFPB004, UFPB77.3, UFPB099 and UFPB134 are promising for ornamental use as potted plant because of their reduced plant height (Table SII). To decrease plant height, the selection of dwarf plants is recommended (Silva Neto et al. 2014, Nascimento et al. 2014, Rêgo et al. 2015, Rêgo and Rêgo 2016, 2018). In addition to plant height, canopy width is also a highly relevant trait to optimize plant growth. Silva Neto et al. (2014) found five or six mean groups with canopy width from 14.0 to $17.0 \mathrm{~cm}$, which is similar to our results. According to Barroso et al. (2011), plants with 22.5 to $26.5 \mathrm{~cm}$ height and 24 to $27 \mathrm{~cm}$ canopy width are desirable as potted ornamental pepper.

\section{GRIFFING}

For almost all characters both additive and nonadditive effects influenced the performance of genotypes (Table I). Additive effects were predominant in the genetic control of hypocotyl diameter, plant height, first bifurcation height, canopy width, leaf length, leaf width and chlorophyll a content. Then, breeders can select for these traits in early segregating generations (Medeiros et al. 2014, 2015, Barroso et al. 2015, 2017, Pessoa et al. 2015). An effective way to decrease or increase these traits could be achieved through repeated backcrosses and selection of desirable recombinants from segregating populations or even by recurrent mass selection that increase the frequency of favorable alleles due to the significant additive gene effects found in this work. 
TABLE III

Estimated general combining ability $(\hat{g} i)$ and specific combining ability $\left(s_{\mathrm{ij}}\right)$ effects for four seedling and plant traits of Capsicum annuum $\mathrm{L}$.

\begin{tabular}{|c|c|c|c|c|}
\hline \multirow{2}{*}{ Accessions (UFPB) } & \multicolumn{4}{|c|}{ Traits } \\
\hline & SH & HD & CL & $\mathbf{C W}$ \\
\hline 001 & $-0.219 * *$ & $0.007 * *$ & $-0.104^{\mathrm{ns}}$ & $-0.007^{\mathrm{ns}}$ \\
\hline 004 & $-0.051^{\mathrm{ns}}$ & $0.009 * *$ & $0.184^{\mathrm{ns}}$ & $0.114 * *$ \\
\hline 77.3 & $0.061^{\mathrm{ns}}$ & $-0.005 * *$ & $-0.123^{\mathrm{ns}}$ & $-0.059 * *$ \\
\hline 099 & $-0.174 * *$ & $-0.003 *$ & $-0.149^{\mathrm{ns}}$ & $0.153 * *$ \\
\hline 134 & $0.409 * *$ & $0.003 *$ & $0.372 * *$ & $-0.056 * *$ \\
\hline 137 & $-0.053^{\mathrm{ns}}$ & $-0.008 * *$ & $0.009^{\text {ns }}$ & $-0.055^{* *}$ \\
\hline 390 & $0.026^{\mathrm{ns}}$ & $-0.002 *$ & $-0.189^{\mathrm{ns}}$ & $-0.090 * *$ \\
\hline
\end{tabular}

Hybrids/Reciprocals (UFPB)

Traits

\begin{tabular}{|c|c|c|c|c|c|c|c|c|}
\hline & \multicolumn{2}{|c|}{ SH } & \multicolumn{2}{|c|}{ HD } & \multicolumn{2}{|c|}{ CL } & \multicolumn{2}{|c|}{ CW } \\
\hline $001 \times 004(004$ x 001) & $0.690 * *$ & $-0.875^{* *}$ & $-0.000^{\mathrm{ns}}$ & $-0.011 * *$ & $0.023^{\mathrm{ns}}$ & $-0.400^{\mathrm{ns}}$ & $-0.083 *$ & $-0.143 * *$ \\
\hline $001 \times 099(099 \times 001)$ & $-0.061^{\mathrm{ns}}$ & $0.003^{\mathrm{ns}}$ & $0.003^{\mathrm{ns}}$ & $0.004^{\mathrm{ns}}$ & $-0.001^{\mathrm{ns}}$ & $0.044^{\mathrm{ns}}$ & $-0.147 * *$ & $0.056^{\mathrm{ns}}$ \\
\hline 004 x $099(099$ x 004) & $0.302 * *$ & $0.594 * *$ & $-0.000^{\mathrm{ns}}$ & $0.011 * *$ & $-0.108^{\mathrm{ns}}$ & $0.325^{\mathrm{ns}}$ & $0.657 * *$ & $0.944 * *$ \\
\hline $77.3 \times 001(001 \times 77.3)$ & $-0.485 * *$ & $-0.687 * *$ & $-0.012 * *$ & $-0.010 * *$ & $-0.138^{\mathrm{ns}}$ & $-0.056^{\mathrm{ns}}$ & $-0.021^{\mathrm{ns}}$ & $-0.081 *$ \\
\hline $77.3 \times 004(004 \times 77.3)$ & $-0.352 * *$ & $-1.075 * *$ & $-0.006^{*}$ & $-0.011 * *$ & $-0.133^{\mathrm{ns}}$ & $-0.400^{\mathrm{ns}}$ & $-0.043^{\mathrm{ns}}$ & $-0.131 * *$ \\
\hline $77.3 \times 099(099 \times 77.3)$ & $0.927 * *$ & $-0.731 * *$ & $0.007 * *$ & $0.004^{\mathrm{ns}}$ & $0.507^{*}$ & $0.219^{\mathrm{ns}}$ & $0.243 * *$ & $0.231 * *$ \\
\hline $134 \times 137(137 \times 134)$ & $0.479 * *$ & $-0.919 * *$ & $-0.006^{*}$ & $-0.001^{\mathrm{ns}}$ & $-0.340^{\mathrm{ns}}$ & $-0.288^{\mathrm{ns}}$ & $0.017^{\mathrm{ns}}$ & $0.025^{\mathrm{ns}}$ \\
\hline $134 \times 390(390 \times 134)$ & $-0.365 * *$ & $0.588 * *$ & $-0.009 * *$ & $0.012 * *$ & $-0.504 *$ & $0.500^{\mathrm{ns}}$ & $-0.010^{\mathrm{ns}}$ & $0.137 * *$ \\
\hline $134 \times 77.3(77.3 \times 134)$ & $0.294 * *$ & $0.494 * *$ & $0.005^{\mathrm{ns}}$ & $0.011 * *$ & $-0.358^{\mathrm{ns}}$ & $0.238^{\mathrm{ns}}$ & $-0.029^{\mathrm{ns}}$ & $0.087 *$ \\
\hline $134 \times 001(001 \times 134)$ & $-0.432 * *$ & $-0.475^{* *}$ & $-0.004^{\mathrm{ns}}$ & $-0.001 * *$ & $-0.121^{\mathrm{ns}}$ & $-0.156^{\mathrm{ns}}$ & $0.043^{\mathrm{ns}}$ & $-0.112 * *$ \\
\hline $134 \times 004(004 \times 134)$ & $-0.069^{\mathrm{ns}}$ & $-1.256^{* *}$ & $0.014 * *$ & $-0.003^{\text {ns }}$ & $1.303 * *$ & $0.619^{*}$ & $0.022^{\mathrm{ns}}$ & $-0.187 * *$ \\
\hline 134 x $099(099 \times 134)$ & $-0.214^{*}$ & $-0.462 * *$ & $-0.009 * *$ & $-0.004^{\mathrm{ns}}$ & $-0.064^{\mathrm{ns}}$ & $-0.094^{\mathrm{ns}}$ & $-0.085^{*}$ & $-0.044^{\mathrm{ns}}$ \\
\hline $137 \times 390(390 \times 137)$ & $-0.241 * *$ & $1.125 * *$ & $0.008 * *$ & $0.012 * *$ & $0.040^{\mathrm{ns}}$ & $0.731 *$ & $-0.024^{\mathrm{ns}}$ & $0.187 * *$ \\
\hline $137 \times 77.3(77.3 \times 390)$ & $0.487 * *$ & $0.800 * *$ & $0.002^{\mathrm{ns}}$ & $0.005^{\mathrm{ns}}$ & $0.199^{\mathrm{ns}}$ & $0.294^{\mathrm{ns}}$ & $-0.023^{\text {ns }}$ & $0.131 * *$ \\
\hline $137 \times 001(001 \times 137)$ & $-0.433 * *$ & $-0.437 * *$ & $0.002^{\mathrm{ns}}$ & $-0.012 * *$ & $-0.127^{\mathrm{ns}}$ & $0.025^{\mathrm{ns}}$ & $-0.064^{\mathrm{ns}}$ & $0.031^{\mathrm{ns}}$ \\
\hline 137 x $004(004$ x 137) & $0.055^{\mathrm{ns}}$ & $-0.906 * *$ & $-0.010 * *$ & $-0.022 * *$ & $-0.146^{\mathrm{ns}}$ & $-0.569 *$ & $0.015^{\mathrm{ns}}$ & $-0.106^{*}$ \\
\hline 137 x $099(099 \times 137)$ & $-0.165^{*}$ & $-1.125 * *$ & $-0.006^{*}$ & $-0.005^{\mathrm{ns}}$ & $0.162^{\mathrm{ns}}$ & $-0.581 *$ & $-0.111 * *$ & $-0.106^{*}$ \\
\hline $390 \times 77.3(77.3 \times 390)$ & $0.082^{\mathrm{ns}}$ & $1.25 * *$ & $0.005^{\mathrm{ns}}$ & $0.016^{* *}$ & $0.123^{\mathrm{ns}}$ & $0.319^{\mathrm{ns}}$ & $-0.134^{\mathrm{ns}}$ & $0.006^{\mathrm{ns}}$ \\
\hline $390 \times 001(001 \times 390)$ & $0.363 * *$ & $-0.562 * *$ & $-0.002^{\mathrm{ns}}$ & $0.002^{\mathrm{ns}}$ & $0.078^{\mathrm{ns}}$ & $-0.031^{\mathrm{ns}}$ & $0.089 *$ & $-0.162^{\mathrm{ns}}$ \\
\hline $390 \times 004(004 \times 390)$ & $-0.024^{\mathrm{ns}}$ & $-1.281 * *$ & $-0.008^{*}$ & $-0.033 * *$ & $-0.010^{\mathrm{ns}}$ & $-0.844 * *$ & $-0.069 *$ & $-0.275^{* *}$ \\
\hline $390 \times 099(099 \times 390)$ & $-0.244 * *$ & $-0.937 * *$ & $-0.002^{\mathrm{ns}}$ & $-0.011 * *$ & $-0.184^{\mathrm{ns}}$ & $-0.325^{\mathrm{ns}}$ & $-0.170 * *$ & $-0.112 * *$ \\
\hline
\end{tabular}


TABLE III (continuation)

\begin{tabular}{|c|c|c|c|c|}
\hline \multirow{2}{*}{ Accessions (UFPB) } & \multicolumn{4}{|c|}{ Traits } \\
\hline & PH & SD & FBH & CAW \\
\hline 001 & $-2.455^{* *}$ & $-0.168^{\mathrm{ns}}$ & $-1.812 * *$ & $-1.916 * *$ \\
\hline 004 & $-0.584^{*}$ & $0.411^{\mathrm{ns}}$ & $-0.899 * *$ & $-0.708 * *$ \\
\hline 77.3 & $3.398 * *$ & $-0.157^{\mathrm{ns}}$ & $2.304 * *$ & $2.945 * *$ \\
\hline 099 & $-0.718 * *$ & $0.425^{\mathrm{ns}}$ & $-1.126 * *$ & $-1.010 * *$ \\
\hline 134 & $-1.705 * *$ & $-0.220^{\mathrm{ns}}$ & $-0.350 * *$ & $-0.528 *$ \\
\hline 137 & $0.193^{\mathrm{ns}}$ & $-0.127^{\mathrm{ns}}$ & $0.453 * *$ & $0.892 * *$ \\
\hline 390 & $1.871 * *$ & $-0.163^{\mathrm{ns}}$ & $1.430 * *$ & $0.325^{\mathrm{ns}}$ \\
\hline
\end{tabular}

Hybrids/Reciprocals (UFPB)

Traits

\begin{tabular}{|c|c|c|c|c|c|c|c|c|}
\hline \multirow[b]{2}{*}{$001 \times 004(004$ x 001) } & \multicolumn{2}{|c|}{ PH } & \multicolumn{2}{|c|}{ SD } & \multicolumn{2}{|c|}{ FBH } & \multicolumn{2}{|c|}{ CAW } \\
\hline & $2.048 *$ & $-1.594 * *$ & $-0.325^{\text {ns }}$ & $-0.029^{\mathrm{ns}}$ & $1.019 * *$ & $-1.094 * *$ & $1.637 * *$ & $-0.969 *$ \\
\hline $001 \times 099(099 \times 001)$ & $0.526^{* *}$ & $0.437^{\mathrm{ns}}$ & $-0.422^{\mathrm{ns}}$ & $-0.004^{\mathrm{ns}}$ & $0.247^{\mathrm{ns}}$ & $-0.156^{\mathrm{ns}}$ & $-0.436^{\mathrm{ns}}$ & $-0.156^{\mathrm{ns}}$ \\
\hline $004 \times 099(099 \times 004)$ & $0.218^{* *}$ & $-0.345^{\mathrm{ns}}$ & $2.929 * *$ & $3.891 * *$ & $0.252^{\mathrm{ns}}$ & $0.875^{* *}$ & $-0.582^{\mathrm{ns}}$ & $-0.781^{\mathrm{ns}}$ \\
\hline $77.3 \times 001(001 \times 77.3)$ & $-1.465^{\mathrm{ns}}$ & $1.437^{*}$ & $0.163^{\mathrm{ns}}$ & $0.071^{\mathrm{ns}}$ & $-0.384^{\mathrm{ns}}$ & $-0.144^{\mathrm{ns}}$ & $-0.454^{\mathrm{ns}}$ & $3.344 * *$ \\
\hline $77.3 \times 004(004 \times 77.3)$ & $-2.679^{\mathrm{ns}}$ & $-1.219 * *$ & $-0.401^{\mathrm{ns}}$ & $-0.012^{\mathrm{ns}}$ & $-1.209 * *$ & $-0.956^{* *}$ & $-1.350 * *$ & $0.156^{\mathrm{ns}}$ \\
\hline $77.3 \times 099(099 \times 77.3)$ & $-0.795^{* *}$ & $-1.094 * *$ & $-0.394^{\mathrm{ns}}$ & $-0.019^{\mathrm{ns}}$ & $-0.119^{\mathrm{ns}}$ & $-0.719 *$ & $0.296^{\mathrm{ns}}$ & -1.125 \\
\hline 134 x $137(137$ x 134) & $-0.510^{\mathrm{ns}}$ & $-1.125^{\mathrm{ns}}$ & $0.142^{\mathrm{ns}}$ & $0.027^{\mathrm{ns}}$ & $-0.544 *$ & $-0.969 * *$ & $-1.289 * *$ & $-1.281 *$ \\
\hline $134 \times 390(390 \times 134)$ & $-0.563^{\mathrm{ns}}$ & $-0.313 * *$ & $0.140^{\mathrm{ns}}$ & $0.019^{\mathrm{ns}}$ & $-0.102^{\mathrm{ns}}$ & $0.075^{\mathrm{ns}}$ & $-0.660^{\mathrm{ns}}$ & $1.844 * *$ \\
\hline $134 \times 77.3(77.3 \times 134)$ & $-0.559^{\mathrm{ns}}$ & $1.219^{*}$ & $0.154^{\mathrm{ns}}$ & $0.008^{\mathrm{ns}}$ & $-0.239^{\mathrm{ns}}$ & $0.750^{*}$ & $-0.186^{\mathrm{ns}}$ & $0.438^{\mathrm{ns}}$ \\
\hline $134 \times 001(001 \times 134)$ & $0.294^{\mathrm{ns}}$ & $0.156^{*}$ & $0.161^{\mathrm{ns}}$ & $0.026^{\mathrm{ns}}$ & $0.108^{\mathrm{ns}}$ & $-0.069^{\mathrm{ns}}$ & $0.769^{\mathrm{ns}}$ & $1.719 * *$ \\
\hline $134 \times 004(004 \times 134)$ & $0.049^{\mathrm{ns}}$ & $0.031 * *$ & $-0.370^{\mathrm{ns}}$ & $0.001^{\mathrm{ns}}$ & $0.214^{\mathrm{ns}}$ & $-1.000 * *$ & $1.873 * *$ & $0.031^{\mathrm{ns}}$ \\
\hline $134 \times 099(099 \times 134)$ & $0.651^{*}$ & $-0.688^{\mathrm{ns}}$ & $-0.394^{\mathrm{ns}}$ & $-0.024^{\mathrm{ns}}$ & $0.210^{\mathrm{ns}}$ & $-0.544^{*}$ & $1.206^{* *}$ & $-0.563^{\mathrm{ns}}$ \\
\hline 137 x $390(390 \times 137)$ & $0.977^{\mathrm{ns}}$ & $0.625 * *$ & $0.195^{\mathrm{ns}}$ & $-0.008^{\mathrm{ns}}$ & $0.519 *$ & $0.000^{\mathrm{ns}}$ & $1.046^{*}$ & $1.469 * *$ \\
\hline $137 \times 77.3(77.3 \times 390)$ & $-1.393^{\mathrm{ns}}$ & $1.281 * *$ & $0.142^{\text {ns }}$ & $0.012^{\mathrm{ns}}$ & $-0.249^{\mathrm{ns}}$ & $1.044 * *$ & $-1.481 * *$ & $0.689^{\mathrm{ns}}$ \\
\hline 137 x 001(001 x 137) & $-0.666^{\mathrm{ns}}$ & $2.031^{\mathrm{ns}}$ & $0.145^{\mathrm{ns}}$ & $0.057^{\mathrm{ns}}$ & $-0.926^{* *}$ & $0.750 *$ & $-0.463^{\mathrm{ns}}$ & $2.906^{* *}$ \\
\hline 137 x $004(004$ x 137) & $-0.224^{\mathrm{ns}}$ & $-0.031^{*}$ & $-0.415^{\mathrm{ns}}$ & $0.023^{\mathrm{ns}}$ & $0.173^{\mathrm{ns}}$ & $-0.850 * *$ & $0.047^{\mathrm{ns}}$ & - \\
\hline 137 x $099(099$ x 137) & $-0.152 * *$ & $-1.406^{*}$ & $-0.409^{\text {ns }}$ & $-0.014^{\mathrm{ns}}$ & $-0.218^{\mathrm{ns}}$ & $-0.231^{\mathrm{ns}}$ & $0.568^{\mathrm{ns}}$ & $-1.281 *$ \\
\hline $390 \times 77.3(77.3 \times 390)$ & $1.772^{\mathrm{ns}}$ & $-0.750^{\mathrm{ns}}$ & $0.176^{\mathrm{ns}}$ & $-0.083^{\text {ns }}$ & $-0.532 *$ & $-0.612 *$ & $1.086^{*}$ & $-1.750 * *$ \\
\hline 390 x $001(001$ x 390) & $-2.188^{*}$ & $-0.875^{* *}$ & $0.083^{\mathrm{ns}}$ & $0.010^{\mathrm{ns}}$ & $-0.653^{*}$ & $-0.188^{\mathrm{ns}}$ & $-1.803^{* *}$ & $-0.688^{n s}$ \\
\hline $390 \times 004(004 \times 390)$ & $0.754^{*}$ & $0.500 * *$ & $-0.392^{\mathrm{ns}}$ & $-0.013^{\text {ns }}$ & $0.627^{*}$ & $-0.306^{\mathrm{ns}}$ & $0.677^{\mathrm{ns}}$ & $-1.313^{*}$ \\
\hline $390 \times 099(099 \times 390)$ & $0.013 * *$ & $1.750 * *$ & $-0.392^{\text {ns }}$ & $0.078^{\mathrm{ns}}$ & $0.099^{\mathrm{ns}}$ & $0.375^{\text {ns }}$ & $0.072^{\mathrm{ns}}$ & $0.531^{\text {ns }}$ \\
\hline
\end{tabular}


TABLE III (continuation)

\begin{tabular}{|c|c|c|c|c|c|c|c|c|}
\hline \multirow{2}{*}{ Accessions (UFPB) } & \multicolumn{8}{|c|}{ Traits } \\
\hline & \multicolumn{2}{|c|}{$\mathbf{L L}$} & \multicolumn{2}{|c|}{$\mathbf{L W}$} & \multicolumn{2}{|c|}{ CLA } & \multicolumn{2}{|c|}{ CLB } \\
\hline 001 & \multicolumn{2}{|c|}{$0.170^{*}$} & \multicolumn{2}{|c|}{$0.129 * *$} & \multicolumn{2}{|c|}{$-0.380^{\mathrm{ns}}$} & \multicolumn{2}{|c|}{$-0.117^{\mathrm{ns}}$} \\
\hline 004 & \multicolumn{2}{|c|}{$0.488 * *$} & \multicolumn{2}{|c|}{$0.246^{* *}$} & \multicolumn{2}{|c|}{$-0.899 *$} & \multicolumn{2}{|c|}{$-0.737^{*}$} \\
\hline 77.3 & \multicolumn{2}{|c|}{$-0.485^{* *}$} & \multicolumn{2}{|c|}{$-0.151^{* *}$} & \multicolumn{2}{|c|}{$-0.076^{\mathrm{ns}}$} & \multicolumn{2}{|c|}{$-0.129^{\mathrm{ns}}$} \\
\hline 099 & \multicolumn{2}{|c|}{$0.391 * *$} & \multicolumn{2}{|c|}{$0.149 * *$} & \multicolumn{2}{|c|}{$-1.521 * *$} & \multicolumn{2}{|c|}{$-0.745 * *$} \\
\hline 134 & \multicolumn{2}{|c|}{$-0.144^{*}$} & \multicolumn{2}{|c|}{$-0.195 * *$} & \multicolumn{2}{|c|}{$3.097 * *$} & \multicolumn{2}{|c|}{$2.064 * *$} \\
\hline 137 & \multicolumn{2}{|c|}{$-0.108^{\mathrm{ns}}$} & \multicolumn{2}{|c|}{$-0.053^{*}$} & \multicolumn{2}{|c|}{$0.200^{\mathrm{ns}}$} & & $35^{\mathrm{ns}}$ \\
\hline 390 & -0.3 & $2 * *$ & -0.1 & $6 * *$ & -0 & $20^{\mathrm{ns}}$ & & $02^{\mathrm{ns}}$ \\
\hline Hybrids/Recinrecals (UFPB) & & & & & iits & & & \\
\hline 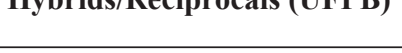 & & $\mathbf{L}$ & & & & $\mathbf{A}$ & & \\
\hline $001 \times 004(004 \times 001)$ & $0.493 * *$ & $-0.408^{*}$ & $0.180^{\mathrm{ns}}$ & $-0.125^{*}$ & $1.392 *$ & $-1.742^{*}$ & $0.842^{\mathrm{ns}}$ & $-0.900^{\mathrm{ns}}$ \\
\hline $001 \times 099(099 \times 001)$ & $-0.162^{\mathrm{ns}}$ & $0.023^{\text {ns }}$ & $-0.039 * *$ & $-0.029^{\mathrm{ns}}$ & $1.775^{*}$ & $2.781 * *$ & $1.202 *$ & $1.839 * *$ \\
\hline 004 x $099(099$ x 004) & $-0.300 *$ & $-0.077^{\mathrm{ns}}$ & $0.037 * *$ & $-0.006^{\mathrm{ns}}$ & $-0.667^{\mathrm{ns}}$ & $-0.696^{\mathrm{ns}}$ & $-0.042^{\mathrm{ns}}$ & $-0.246^{\mathrm{ns}}$ \\
\hline $77.3 \times 001(001 \times 77.3)$ & $-0.125^{\text {ns }}$ & $0.171^{\mathrm{ns}}$ & $-0.084^{\mathrm{ns}}$ & $0.068^{\mathrm{ns}}$ & $-1.749 *$ & $-0.702^{\mathrm{ns}}$ & $-1.446^{*}$ & $-0.121^{\mathrm{ns}}$ \\
\hline $77.3 \times 004(004 \times 77.3)$ & $-0.317^{*}$ & $-0.538 * *$ & $-0.070^{\mathrm{ns}}$ & $-0.224 * *$ & $0.079^{\mathrm{ns}}$ & $0.209^{\mathrm{ns}}$ & $0.287^{\mathrm{ns}}$ & $-0.329^{n s}$ \\
\hline 77.3 x $099(099 \times 77.3)$ & $0.012^{\mathrm{ns}}$ & $-0.346^{*}$ & $0.011^{\mathrm{ns}}$ & $-0.079^{\mathrm{ns}}$ & $-1.043^{\mathrm{ns}}$ & $0.946^{\mathrm{ns}}$ & $-0.971^{\mathrm{ns}}$ & $0.383^{\mathrm{ns}}$ \\
\hline $134 \times 137(137 \times 134)$ & $-0.316^{*}$ & $0.139^{\text {ns }}$ & $-0.036^{\mathrm{ns}}$ & $-0.002^{\mathrm{ns}}$ & $-0.690^{\mathrm{ns}}$ & $0.485^{\text {ns }}$ & $-0.272^{\mathrm{ns}}$ & $-0.187^{\mathrm{ns}}$ \\
\hline $134 \times 390(390 \times 134)$ & $-0.097^{\mathrm{ns}}$ & $0.200^{\mathrm{ns}}$ & $-0.033^{\mathrm{ns}}$ & $0.065^{\text {ns }}$ & $1.509 *$ & $-1.373^{\mathrm{ns}}$ & $0.817^{\mathrm{ns}}$ & $-1.400^{*}$ \\
\hline $134 \times 77.3(77.3 \times 134)$ & $-0.127^{\mathrm{ns}}$ & $0.379 *$ & $-0.060^{\mathrm{ns}}$ & $0.067^{\text {ns }}$ & $-0.051^{\mathrm{ns}}$ & $-0.348^{\mathrm{ns}}$ & $-0.588^{\mathrm{ns}}$ & $-0.207^{\mathrm{ns}}$ \\
\hline 134 x $001(001$ x 134) & $0.031^{\mathrm{ns}}$ & $-0.035^{\mathrm{ns}}$ & $-0.002^{\mathrm{ns}}$ & $0.025^{\mathrm{ns}}$ & $0.634^{\mathrm{ns}}$ & $0.554^{\mathrm{ns}}$ & $0.178^{\mathrm{ns}}$ & $-0.267^{\mathrm{ns}}$ \\
\hline 134 x $004(004 \times 134)$ & $0.064^{\mathrm{ns}}$ & $-0.198^{\mathrm{ns}}$ & $0.035^{\mathrm{ns}}$ & $0.017^{\mathrm{ns}}$ & $-0.743^{\mathrm{ns}}$ & $-1.65^{*}$ & $-0.587^{\mathrm{ns}}$ & $-0.460^{\mathrm{ns}}$ \\
\hline 134 x $099(099 \times 134)$ & $0.138^{\mathrm{ns}}$ & $0.229^{\mathrm{ns}}$ & $0.034^{\mathrm{ns}}$ & $0.056^{\mathrm{ns}}$ & $-0.035^{\mathrm{ns}}$ & $-1.385^{\mathrm{ns}}$ & $0.796^{\mathrm{ns}}$ & $-2.206^{* *}$ \\
\hline 137 x $390(390 \times 137)$ & $0.227^{\mathrm{ns}}$ & $0.006^{\mathrm{ns}}$ & $0.105^{\text {ns }}$ & $-0.129 *$ & $1.999 *$ & $-3.589 * *$ & $1.270^{*}$ & $-2.538^{*}$ \\
\hline $137 \times 77.3(77.3 \times 390)$ & $-0.074^{\mathrm{ns}}$ & $0.331^{\mathrm{ns}}$ & $-0.022^{\mathrm{ns}}$ & $0.052 *$ & $-1.142^{\mathrm{ns}}$ & $-1.356^{\mathrm{ns}}$ & $-1.094 *$ & $-0.412^{\mathrm{ns}}$ \\
\hline 137 x 001(001 x 137) & $-0.020^{\mathrm{ns}}$ & $0.554^{*}$ & $-0.019^{\mathrm{ns}}$ & $0.160^{\mathrm{ns}}$ & $-1.125^{\mathrm{ns}}$ & $-1.631^{*}$ & $-0.648^{n s}$ & $-0.713^{n s}$ \\
\hline 137 x $004(004$ x 137) & $0.075^{\mathrm{ns}}$ & $0.033^{\mathrm{ns}}$ & $-0.046^{\mathrm{ns}}$ & $0.017^{\mathrm{ns}}$ & $0.374^{\mathrm{ns}}$ & $0.133^{\mathrm{ns}}$ & $-0.221^{\mathrm{ns}}$ & $0.419^{\mathrm{ns}}$ \\
\hline 137 x $099(099 \times 137)$ & $0.141^{\mathrm{ns}}$ & $-0.098^{\mathrm{ns}}$ & $-0.001^{\mathrm{ns}}$ & $-0.098^{\mathrm{ns}}$ & $-0.101^{\mathrm{ns}}$ & $1.131^{\mathrm{ns}}$ & $-0.217^{\mathrm{ns}}$ & $0.373^{\mathrm{ns}}$ \\
\hline $390 \times 77.3(77.3 \times 390)$ & $0.089^{\mathrm{ns}}$ & $-0.348^{*}$ & $-0.028^{\mathrm{ns}}$ & $-0.115^{\mathrm{ns}}$ & $-0.045^{\text {ns }}$ & $-0.067^{\mathrm{ns}}$ & $-0.517^{\mathrm{ns}}$ & $0.006^{\mathrm{ns}}$ \\
\hline $390 \times 001(001 \times 390)$ & $-0.324 *$ & $-0.338^{\mathrm{ns}}$ & $-0.029^{\mathrm{ns}}$ & $0.152^{*}$ & $-0.859^{\mathrm{ns}}$ & $2.639 * *$ & $0.643^{\mathrm{ns}}$ & $2.844 * *$ \\
\hline $390 \times 004(004$ x 390) & $0.251^{\mathrm{ns}}$ & $-0.575^{* *}$ & $0.160 * *$ & $-0.075^{\mathrm{ns}}$ & $0.061^{\mathrm{ns}}$ & $1.634^{*}$ & $-0.154^{\mathrm{ns}}$ & $1.289^{*}$ \\
\hline $390 \times 099(099 \times 390)$ & $-0.084^{\mathrm{ns}}$ & $-0.173^{\mathrm{ns}}$ & $-0.070^{\mathrm{ns}}$ & $0.010^{\mathrm{ns}}$ & $-1.298^{\mathrm{ns}}$ & $0.164^{\mathrm{ns}}$ & $-0.825^{\mathrm{ns}}$ & $0.756^{\mathrm{ns}}$ \\
\hline
\end{tabular}

${ }^{n s}$ Not significant $* *$ and* Significant according to the Student's t-test, at $\mathrm{p} \leq 0.01$ and $\mathrm{p} \leq 0.05$, respectively. SH - Seedling height; HD - Hypocotyl diameter; CL - Cotyledon leaf length; CW - Cotyledon leaf width; PH - Plant height; SD - Stem diameter; FBH First Bifurcation Height; CAW - Canopy width; LL - Leaf length; LW - Leaf width; CLA - Chlorophyll a and CLB - Chlorophyll b. 
The non-additive gene effects (dominance and/or epistasis) played a more important role than additive effects in seedling height, cotyledonous leaf length and width and chlorophyll b content (Table I). Ferreira et al. (2015) reported similar results for seedling traits and these results suggest the possibility of vigor hybrid exploitation because of the significant non-additive effects found. Except for cotyledonous leaf length and stem diameter, all traits showed reciprocal effects, demonstrating differences when a given genotype is used as male or female. This fact indicated that maternal effect genes may be involved in the genetic control of these traits and that improvements may be obtained by inverting the parent cross order. This can also be due to genetic cytoplasmic factors, as found earlier (Rêgo et al. 2009, Rêgo and Rêgo 2016).

Some parents are more promising than others for the development of superior populations. Based on estimates for the combining ability effects genitor UFPB134 must be involved in the hybrid combinations for seedling traits and parents UFPB001 and UFPB099 must compose the hybrid combinations for improving plant traits. Desirable recombinants can be selected in segregating populations, which increase the frequency of favorable alleles (Silva Neto et al. 2014, Nascimento et al. 2014, Rêgo et al. 2015, Rêgo and Rêgo 2016, 2018). According to Rêgo et al. (2009), good general combiners showed better mean performance, indicating that the parent may be selected either on the basis of GCA, mean performance or by combination of them. Afterwards, crosses showing desirable $\mathrm{S}_{\mathrm{ij}}$ and a parent with a good general combining ability should be used in breeding programs for potted ornamental peppers (Table III).

Leaf traits (length and width) are important for a harmonious plant shape and the hybrids UFPB134 x UFPB137, UFPB134 x UFPB390, UFPB134 x UFPB77.3, UFPB137 x UFPB134, UFPB390 x UFPB134, UFPB390 x UFPB77.3,
UFPB77.3 x UFPB134 and UFPB77.3 x UFPB137 are indicated for selection. Similar results were found by Nascimento et al. (2012) for leaf traits of ornamental pepper, and they suggested that the genotypes presenting the smallest leaves should be preferred. Parents and hybrids that present higher leaf chlorophyll $\mathrm{a}$ and $\mathrm{b}$ concentrations are indicated for selection because the plant growth and adaptability to different environments are closely related to light capture and photosynthesis. Pessoa et al. (2018) found different contents for chlorophyll $\mathrm{a}$ and $\mathrm{b}$ among genotypes in ornamental peppers, suggesting the selection of plants with higher levels of chlorophyll.

\section{GARDNER AND EBERHART}

Heterosis was significant for all traits, except for leaf length and stem diameter. Besides this, specific heterosis was significant for all traits excepting cotyledonous leaf length, leaf length and width, and chlorophyll $\mathrm{a}$ and $\mathrm{b}$ contents and then it is possible to explore heterosis or heterobeltiosis (Table IV). Significant specific heterosis indicates that nonadditive effects on trait expression are important. Non-additive effects were also predominant for seedling traits as highlighted by the Griffing diallel analysis. Significant specific heterosis can indicate complementation between pairs of parents in crosses and this helps to improve the performance of specific hybrids (Oliveira et al. 2014, Benin et al. 2009).

Fast-growing pepper seedlings may be transplanted earlier, decreasing production costs and time (Barroso et al. 2012). Less vigorous plants can lead to death and affect the plant stand causing profit loss to nursery owners. However, less vigorous seedling will not affect the plant traits since there was no genotypic correlation among seedling traits and plant height (data not shown). In addition to differences in seedling traits, different genotypes showed differences in all traits evaluated, indicating 
TABLE IV

Heterosis $(\mathrm{H})$ and heterobeltiosis $(\mathrm{Hb})$ for seedling and plant traits of Capsicum annuum $\mathrm{L}$.

\begin{tabular}{|c|c|c|c|c|c|c|c|c|}
\hline \multirow{2}{*}{ Hybrids (UFPB) } & \multicolumn{2}{|c|}{$\mathrm{SH}(\mathrm{cm})$} & \multicolumn{2}{|c|}{ HD (cm) } & \multicolumn{2}{|c|}{ CL } & \multicolumn{2}{|c|}{ CW } \\
\hline & $\mathbf{H}$ & Hb & H & Hb & $\mathbf{H}$ & $\mathbf{H b}$ & $\mathbf{H}$ & $\mathbf{H b}$ \\
\hline $001 \times 134$ & -16.12 & -31.62 & -3.73 & -7.19 & -4.80 & -15.00 & 4.99 & -7.50 \\
\hline $001 \times 137$ & -13.84 & -21.12 & 0.73 & -9.80 & -14.16 & -16.38 & -29.39 & -32.50 \\
\hline $001 \times 390$ & 13.39 & 5.88 & -10.25 & -16.99 & -9.56 & -9.56 & 6.70 & -1.30 \\
\hline $001 \times 77.3$ & 15.09 & 3.39 & -5.97 & -17.65 & -5.04 & -14.11 & 3.12 & -17.50 \\
\hline $001 \times 004$ & -1.85 & -10.17 & -14.93 & -15.48 & -2.30 & -13.64 & -8.83 & -28.80 \\
\hline $001 \times 099$ & 0.94 & -10.17 & -1.79 & -10.46 & 2.33 & -10.47 & 1.37 & -11.30 \\
\hline $004 \times 134$ & 19.21 & -9.19 & 5.05 & 0.64 & 39.42 & 11.77 & 66.16 & 44.36 \\
\hline $004 \times 137$ & 23.35 & 4.24 & 0.00 & -10.97 & 31.16 & 13.34 & 37.31 & 10.96 \\
\hline $004 \times 390$ & 36.76 & 17.65 & 10.88 & 1.93 & 43.97 & 27.27 & 50.42 & 24.94 \\
\hline $004 \times 77.3$ & 50.02 & 46.96 & 0.00 & -12.90 & 38.34 & 34.83 & 67.81 & 62.50 \\
\hline $004 \times 001$ & 50.01 & 37.29 & -1.30 & -1.93 & 30.60 & 15.45 & 28.04 & 0.00 \\
\hline 004 x 099 & 49.47 & 44.90 & 1.78 & -7.74 & 40.15 & 38.49 & 311.58 & 260.00 \\
\hline $77.3 \times 134$ & -8.11 & -30.99 & -8.17 & -16.90 & -18.78 & -33.57 & -11.89 & -21.26 \\
\hline $77.3 \times 137$ & -6.10 & -21.97 & -6.78 & -9.09 & -3.92 & -15.10 & -25.66 & -38.38 \\
\hline $77.3 \times 390$ & -25.23 & -36.78 & -12.65 & -17.69 & -13.08 & -21.38 & -8.69 & -22.12 \\
\hline $77.3 \times 001$ & -26.42 & -33.90 & -20.90 & -30.72 & -9.55 & -18.18 & -17.25 & -33.80 \\
\hline $77.3 \times 004$ & -21.65 & -23.25 & -16.30 & -27.09 & 1.45 & -1.12 & 22.55 & 18.67 \\
\hline $77.3 \times 099$ & 32.48 & 31.09 & 7.88 & 13.04 & 45.79 & 40.45 & 107.41 & 86.67 \\
\hline $099 \times 134$ & -2.58 & -27.35 & -8.95 & -14.08 & 5.18 & -16.43 & 17.33 & 16.40 \\
\hline $099 \times 137$ & 23.08 & 1.42 & -8.50 & -10.32 & 32.00 & 12.93 & 11.31 & 1.42 \\
\hline $099 \times 390$ & 21.04 & 1.46 & 1.56 & 0.00 & 2.87 & -10.00 & 4.62 & -1.53 \\
\hline $099 \times 77.3$ & 82.79 & 80.86 & 0.41 & -3.97 & 25.36 & 20.76 & 38.81 & 24.93 \\
\hline $099 \times 001$ & 0.94 & -10.17 & -6.81 & -15.03 & -1.29 & -13.64 & -11.43 & -22.50 \\
\hline $099 \times 004$ & 9.48 & 6.14 & -13.88 & -21.93 & 9.01 & 7.72 & 23.78 & 8.27 \\
\hline $134 \times 137$ & -47.02 & -53.42 & -14.07 & -20.42 & -24.22 & -30.71 & -14.93 & -21.93 \\
\hline $134 \times 390$ & -12.38 & -24.36 & -4.41 & -8.45 & 23.20 & 10.00 & 0.74 & -4.47 \\
\hline $134 \times 77.3$ & 14.37 & -14.10 & 8.95 & -1.41 & -2.18 & -20.00 & 13.80 & 1.71 \\
\hline $134 \times 001$ & -36.04 & -47.86 & -17.29 & -20.26 & -14.82 & -23.94 & -20.54 & -30.00 \\
\hline $134 \times 004$ & -37.16 & -52.14 & 1.01 & -3.22 & 83.53 & 47.14 & 9.52 & -4.86 \\
\hline $134 \times 099$ & -23.78 & -43.16 & -15.67 & -20.42 & -1.58 & -21.80 & 5.82 & 4.99 \\
\hline $137 \times 134$ & -11.31 & -22.02 & -11.79 & -18.31 & -6.25 & -14.29 & -8.96 & -16.45 \\
\hline $137 \times 390$ & 6.48 & 4.24 & 8.37 & 4.61 & 15.47 & 12.48 & -3.52 & -6.79 \\
\hline $137 \times 77.3$ & 37.29 & 14.09 & 1.69 & -0.83 & 19.02 & 5.17 & 9.13 & -9.54 \\
\hline $137 \times 001$ & -35.38 & -40.84 & -16.06 & -24.83 & -12.39 & -14.65 & -22.91 & -26.3 \\
\hline $137 \times 004$ & -25.00 & -36.62 & -31.16 & -38.70 & -14.21 & -25.86 & 8.55 & -12.28 \\
\hline $137 \times 099$ & -38.46 & -49.29 & -16.60 & -18.25 & -14.87 & -27.17 & -14.32 & -21.93 \\
\hline $390 \times 134$ & -35.64 & -44.44 & -22.79 & -26.06 & -40.80 & -47.14 & -33.37 & -36.82 \\
\hline $390 \times 137$ & -45.32 & -46.47 & -11.55 & -14.61 & -36.28 & -37.93 & -46.08 & -47.92 \\
\hline $390 \times 77.3$ & 44.34 & 22.05 & 14.29 & 7.69 & 12.56 & 1.82 & -6.89 & -20.59 \\
\hline $390 \times 001$ & -14.95 & -20.59 & -7.42 & -14.37 & -11.82 & -11.82 & -28.43 & -33.80 \\
\hline $390 \times 004$ & -33.34 & -42.66 & -35.44 & -40.64 & -25.46 & -34.11 & -27.48 & -39.76 \\
\hline $390 \times 099$ & -31.59 & -42.66 & -15.62 & -16.92 & -24.15 & -33.64 & -23.50 & -28.00 \\
\hline
\end{tabular}


TABLE IV (continuation)

\begin{tabular}{|c|c|c|c|c|c|c|c|c|}
\hline \multirow{2}{*}{ Hybrids (UFPB) } & \multicolumn{2}{|c|}{ PH (cm) } & \multicolumn{2}{|c|}{$\mathrm{SD}(\mathrm{cm})$} & \multicolumn{2}{|c|}{ FBH (cm) } & \multicolumn{2}{|c|}{ CAW (cm) } \\
\hline & $\mathbf{H}$ & $\mathbf{H b}$ & H & Hb & H & $\mathbf{H b}$ & $\mathbf{H}$ & $\mathbf{H b}$ \\
\hline $001 \times 134$ & -5.29 & -3.40 & -8.05 & -17.51 & -4.29 & 19.09 & -1.93 & -1.29 \\
\hline $001 \times 137$ & -22.29 & -8.95 & -15.93 & -8.95 & -32.04 & 0.00 & -16.61 & -5.45 \\
\hline $001 \times 390$ & -8.27 & 9.18 & -19.15 & 9.18 & -9.23 & 39.76 & -4.98 & 1.55 \\
\hline $001 \times 77.3$ & -25.21 & 8.81 & -13.35 & 8.81 & -17.78 & 59.76 & -17.63 & 1.30 \\
\hline $001 \times 004$ & -1.08 & 5.11 & 9.77 & 5.11 & 3.03 & 4.54 & 6.08 & 7.52 \\
\hline $001 \times 099$ & 2.62 & 7.33 & -9.61 & 7.33 & 0.55 & 3.40 & -1.69 & -1.56 \\
\hline $004 \times 134$ & -1.19 & 2.85 & 10.59 & 2.85 & 22.75 & 50.12 & 16.11 & 18.46 \\
\hline $004 \times 137$ & -5.25 & 3.95 & -3.78 & 3.95 & 11.47 & 60.93 & 1.53 & 16.86 \\
\hline $004 \times 390$ & 3.39 & 15.13 & 5.35 & 15.13 & 16.97 & 76.62 & 13.19 & 22.73 \\
\hline $004 \times 77.3$ & -15.37 & 14.14 & 7.10 & 14.14 & -10.04 & 71.09 & -4.78 & 18.99 \\
\hline $004 \times 001$ & 16.68 & 23.98 & 18.64 & 23.98 & 42.21 & 44.31 & 14.24 & 15.79 \\
\hline $004 \times 099$ & 2.84 & 4.41 & 29.21 & 4.41 & 33.12 & 34.88 & 1.47 & 2.72 \\
\hline $77.3 \times 134$ & -18.69 & 15.31 & -2.77 & -11.93 & -21.04 & 16.04 & -2.73 & 18.72 \\
\hline $77.3 \times 137$ & -22.64 & -6.33 & -7.21 & -13.45 & -24.70 & -6.43 & -12.19 & -5.53 \\
\hline $77.3 \times 390$ & 1.24 & 20.65 & 12.13 & 9.77 & -9.56 & 7.98 & 6.40 & 21.46 \\
\hline $77.3 \times 001$ & -13.51 & 25.84 & 8.69 & 7.53 & -20.47 & 54.54 & 4.96 & 29.09 \\
\hline $77.3 \times 004$ & -24.88 & 1.32 & 3.18 & -0.78 & -27.79 & 37.32 & -3.72 & 20.33 \\
\hline $77.3 \times 099$ & -16.64 & 14.57 & -5.00 & -10.65 & -18.15 & 52.70 & -4.44 & 17.71 \\
\hline $099 \times 134$ & 6.95 & 9.61 & 0.97 & 9.61 & 11.61 & -34.41 & 13.18 & 14.06 \\
\hline $099 \times 137$ & 2.43 & 14.24 & -5.00 & 14.24 & -4.54 & 35.49 & 5.96 & 20.31 \\
\hline $099 \times 390$ & -5.39 & 7.12 & -16.14 & 7.12 & -0.72 & 47.31 & 1.22 & 8.33 \\
\hline $099 \times 77.3$ & -8.02 & 26.44 & 0.44 & 26.44 & -4.90 & 77.42 & 3.17 & 27.08 \\
\hline $099 \times 001$ & -2.33 & 2.15 & -8.29 & 2.15 & 6.08 & 9.09 & -0.39 & -0.52 \\
\hline $099 \times 004$ & 2.84 & 4.41 & 1.45 & 4.41 & 2.61 & 3.97 & 8.06 & 9.39 \\
\hline $134 \times 137$ & -14.59 & -2.13 & -2.31 & -16.84 & -24.49 & -12.97 & -9.11 & 2.31 \\
\hline $134 \times 390$ & -3.97 & 11.75 & -4.06 & -22.96 & -2.39 & 16.98 & 8.69 & 15.38 \\
\hline $134 \times 77.3$ & -8.91 & 29.18 & -0.17 & -9.57 & -8.57 & 34.36 & 0.21 & 22.30 \\
\hline $134 \times 001$ & -3.48 & -1.55 & 1.03 & -9.37 & -6.30 & 16.58 & 12.26 & 12.99 \\
\hline $134 \times 004$ & -0.85 & 3.21 & 10.95 & 4.25 & -6.13 & 14.80 & 16.37 & 18.73 \\
\hline $134 \times 099$ & -0.69 & 1.78 & -6.77 & -20.05 & -3.92 & 15.71 & 8.53 & 9.37 \\
\hline $137 \times 134$ & -3.42 & 10.68 & -10.93 & -24.18 & -3.97 & 10.69 & 0.23 & 12.82 \\
\hline $137 \times 390$ & 4.35 & 5.79 & -1.92 & -6.66 & -1.13 & 2.34 & 6.69 & 12.78 \\
\hline $137 \times 77.3$ & -2.39 & 18.18 & -3.71 & -10.19 & -8.99 & 13.10 & -8.00 & -1.02 \\
\hline $137 \times 001$ & -1.77 & 15.10 & 0.50 & -5.30 & -13.51 & 27.27 & 4.69 & 18.70 \\
\hline $137 \times 004$ & -5.55 & 3.61 & 3.17 & -7.20 & -9.33 & 30.91 & 1.53 & 16.86 \\
\hline $137 \times 099$ & -11.24 & -1.01 & -8.70 & -9.51 & -10.15 & 27.53 & -3.44 & 9.63 \\
\hline $390 \times 134$ & -0.91 & 15.30 & -10.66 & -20.60 & -3.82 & 15.27 & -5.56 & 0.25 \\
\hline $390 \times 137$ & -1.08 & 0.28 & 0.35 & -4.48 & -1.13 & 2.34 & -3.46 & 2.05 \\
\hline $390 \times 77.3$ & -4.16 & 14.21 & -13.52 & -15.34 & -18.53 & -2.73 & -4.80 & 8.68 \\
\hline $390 \times 001$ & -16.98 & -1.18 & -16.11 & -16.99 & -13.66 & 32.95 & -10.33 & -4.16 \\
\hline $390 \times 004$ & 8.12 & 20.39 & 1.04 & -4.81 & 9.79 & 65.79 & 2.85 & 11.53 \\
\hline $390 \times 099$ & 11.38 & 26.10 & 6.34 & 2.07 & 7.97 & 60.22 & 5.35 & 12.76 \\
\hline
\end{tabular}


TABLE IV (continuation)

\begin{tabular}{|c|c|c|c|c|c|c|c|c|}
\hline \multirow{2}{*}{ Hybrids (UFPB) } & \multicolumn{2}{|c|}{ LL (cm) } & \multicolumn{2}{|c|}{ LW (cm) } & \multicolumn{2}{|c|}{ CLA } & \multicolumn{2}{|c|}{ CLB } \\
\hline & $\mathbf{H}$ & $\mathbf{H b}$ & $\mathbf{H}$ & $\mathbf{H b}$ & $\mathbf{H}$ & $\mathbf{H b}$ & $\mathbf{H}$ & $\mathbf{H b}$ \\
\hline $001 \times 134$ & -2.19 & 1.22 & -2.89 & 14.56 & 1.28 & -7.61 & 9.49 & -10.75 \\
\hline $001 \times 137$ & -9.82 & -4.46 & -9.27 & -0.92 & 0.64 & -2.38 & -1.55 & -10.91 \\
\hline $001 \times 390$ & -0.14 & -8.76 & -6.71 & 11.43 & -9.48 & -11.56 & -15.30 & -18.58 \\
\hline $001 \times 77.3$ & -10.13 & -3.21 & -13.58 & -6.67 & -9.23 & -15.29 & -29.01 & -42.62 \\
\hline $001 \times 004$ & 2.46 & 4.51 & 9.62 & 11.04 & -0.227 & -2.66 & 4.95 & 1.13 \\
\hline $001 \times 099$ & -4.68 & -0.38 & -3.59 & -1.93 & 13.19 & 11.59 & 42.75 & 38.99 \\
\hline $004 \times 134$ & 3.72 & 9.57 & 7.26 & 24.68 & 4.52 & -6.76 & 1.05 & -19.95 \\
\hline $004 \times 137$ & 3.02 & 11.44 & 3.82 & 11.81 & 0.49 & -4.83 & -13.25 & -24.06 \\
\hline $004 \times 390$ & 16.14 & 31.07 & 23.66 & 45.53 & -2.23 & -2.38 & -10.13 & -10.36 \\
\hline $004 \times 77.3$ & 1.31 & 11.45 & 8.74 & 15.84 & -5.87 & -14.14 & -14.29 & -32.64 \\
\hline $004 \times 001$ & 14.66 & 16.96 & 21.26 & 22.83 & 11.66 & 8.93 & 27.84 & 23.18 \\
\hline $004 \times 099$ & -5.33 & -3.04 & 7.55 & 10.84 & -6.22 & -7.22 & -3.33 & -4.37 \\
\hline $77.3 \times 134$ & -15.75 & -18.81 & -16.32 & -9.12 & -3.84 & -6.18 & -18.13 & -19.01 \\
\hline $77.3 \times 137$ & -11.43 & -10.40 & -11.36 & -9.06 & -6.31 & -9.98 & -29.49 & -37.90 \\
\hline $77.3 \times 390$ & 3.53 & 5.89 & 0.72 & 10.66 & -4.01 & -12.32 & -19.95 & -37.20 \\
\hline $77.3 \times 001$ & -4.56 & 2.78 & -6.91 & 0.53 & -13.57 & -19.34 & -31.28 & -44.45 \\
\hline $77.3 \times 004$ & -15.09 & -7.47 & -13.64 & -8.00 & -4.54 & -12.93 & -20.61 & -37.61 \\
\hline $77.3 \times 099$ & -11.42 & 0.00 & -10.11 & -1.12 & -8.63 & -15.84 & -26.52 & -41.80 \\
\hline $099 \times 134$ & -5.62 & 2.23 & -3.48 & 16.12 & 2.98 & -7.25 & 30.42 & 4.15 \\
\hline $099 \times 137$ & 1.95 & 13.14 & 3.55 & 15.19 & -7.39 & -11.39 & -13.59 & -23.65 \\
\hline $099 \times 390$ & -0.13 & 15.73 & -2.20 & 15.16 & -5.06 & -5.92 & -13.03 & -14.17 \\
\hline $099 \times 77.3$ & -0.65 & 12.16 & -2.45 & 7.31 & -14.56 & -21.30 & -33.84 & -47.59 \\
\hline $099 \times 001$ & -5.35 & -1.08 & -0.97 & 0.73 & -5.59 & -6.92 & -3.54 & -6.08 \\
\hline $099 \times 004$ & -3.14 & -0.80 & 8.10 & 11.41 & -1.40 & -2.45 & 3.09 & 1.99 \\
\hline $134 \times 137$ & -5.21 & -3.05 & -4.51 & 2.56 & -0.68 & -6.79 & -7.62 & -17.86 \\
\hline $134 \times 390$ & -0.32 & 6.13 & 3.41 & 4.53 & 3.48 & -7.56 & 2.00 & -19.34 \\
\hline $134 \times 77.3$ & -2.93 & 0.88 & -8.66 & -0.81 & -5.79 & -8.09 & -21.28 & -22.13 \\
\hline $134 \times 001$ & -3.32 & 0.06 & -0.24 & 17.68 & 4.62 & -4.56 & 4.44 & -14.86 \\
\hline $134 \times 004$ & -2.38 & 3.13 & 9.09 & 26.81 & -5.63 & -15.82 & -7.93 & -27.07 \\
\hline $134 \times 099$ & 1.29 & 9.72 & 2.34 & 23.12 & -5.46 & -14.86 & -12.24 & -29.92 \\
\hline $137 \times 134$ & -9.86 & -7.80 & -4.28 & 2.81 & -3.52 & -9.46 & -4.38 & -14.98 \\
\hline $137 \times 390$ & 4.98 & 50.00 & 1.09 & 9.83 & -4.12 & -9.06 & -14.11 & -24.97 \\
\hline $137 \times 77.3$ & 0.04 & 1.61 & -5.71 & -4.73 & -14.46 & -17.80 & -36.57 & -44.13 \\
\hline $137 \times 001$ & 8.01 & 14.42 & 6.73 & 16.54 & -9.89 & -12.58 & -17.18 & -25.05 \\
\hline $137 \times 004$ & 4.07 & 12.58 & 5.48 & 13.61 & 1.37 & -3.99 & -3.76 & -15.76 \\
\hline $137 \times 099$ & -1.06 & 9.80 & -6.04 & 4.51 & 0.01 & -4.30 & -5.22 & -16.25 \\
\hline $390 \times 134$ & -7.25 & -1.23 & -4.74 & -3.70 & 11.92 & -0.02 & 29.35 & 2.28 \\
\hline $390 \times 137$ & 4.75 & 8.97 & 16.31 & 26.37 & 19.56 & 13.40 & 43.54 & 25.38 \\
\hline $390 \times 77.3$ & -8.99 & -6.83 & -12.58 & -3.96 & -4.43 & -12.70 & -19.82 & -37.11 \\
\hline $390 \times 001$ & -11.40 & -2.15 & 9.59 & 30.91 & 8.51 & 6.02 & 57.21 & 51.11 \\
\hline $390 \times 004$ & -2.63 & 9.89 & 15.52 & 35.95 & 9.18 & 9.02 & 24.03 & 23.72 \\
\hline $390 \times 099$ & -5.63 & 9.36 & -1.10 & 20.49 & -3.92 & -4.79 & 6.80 & 5.39 \\
\hline
\end{tabular}

SH - Seedling height; HD - Hypocotyl diameter; CL - Cotyledonous leaf length; CW - Cotyledonous leaf width; PH - Plant height; SD - Stem diameter; FBH - Height of first branching; CAW - Canopy width; LL - Leaf length; LW - Leaf width; CLA - Chlorophyll a; CLB - Chlorophyll b. 
the possibility of selecting them (parents and hybrids) according to traits of interest. Differences in heterosis and parent effects on plant traits were also observed among genotypes. This variability is essential to plant breeders because there is no plant improvement without it. Variability enables the selection of superior genotypes (Rocha et al. 2014) and heterosis (hybrid vigor) has been extensively explored for the improvement of several crops and development of new populations through crosses (Mendes et al. 2015, Rêgo et al. 2009, Rêgo and Rêgo 2016, 2018). According to Nascimento et al. (2011) higher negative heterosis and heterobeltiosis for plant size in ornamental pepper are desirable since the hybrids will be smaller than their parents means. Beside this, dwarf plants are indicated for ornamental purposes because they are ideal for growth in pots (Barroso et al. 2012, Rêgo et al. 2009, Rêgo and Rêgo 2016, 2018). Rêgo and Rêgo $(2016,2018)$ related that pepper could be grown in either pots or gardens, with small plants being recommended for cultivation in pots and large ones for exterior environments as gardens. In addition, ornamental pepper for growth in pots should have small leaves, which turns flowers and fruits more visible, making potted pepper plants more attractive to the consumer (Ferreira et al. 2015).

\section{CONCLUSION}

Crosses showing desirable $\mathrm{S}_{\mathrm{ij}}$ and a parent with a good general combining ability should be used in breeding programs to potted ornamental peppers. The Griffing diallel analysis showed that the genitor 134 was a good parent with positive GCA effects for seedling traits and the parent with major values of $g_{i}$ for cotyledonous leaf width was the UFPB099. Therefore, both genotypes should compose the hybrids to continue improving seedling traits in a breeding program. Genitors UFPB134, UFPB 001 and UFPB099 are indicated to decrease plant size as they are involved in almost all hybrids combinations with the best SCA values. Based on these findings, hybrids UFPB390 x UFPB001, UFPB004 x UFPB001, UFPB004 x UFPB099 and UFPB004 x UFPB77.3 can be used as new hybrid cultivars. In fact, additive effects were important in the genetic control of plant traits and can used for improving C. annuum. Recurrent selection or mass selection might lead to releasing new varieties with reduced plant size. For those traits in which nonadditive variances were important (seedling traits), there was an additional opportunity for developing $F_{1}$ hybrid with high heterobeltiosis. Finally, our results clearly show potted ornamental pepper varieties can be developed through hybrid breeding in $C$. annuum.

\section{AUTHOR CONTRIBUTIONS}

Elizanilda Ramalho do Rêgo, Angela Maria dos S. Pessoa and Maílson M. do Rêgo designed the field research, made the genetic analysis and performed the statistical analysis. Angela Maria dos Santos Pessoa, Cristine Agrine P. dos Santos, Michelle G. de Carvalho and Julio Carlos P. de Mesquita developed the search on the field.

Angela Maria dos S. Pessoa and Elizanilda Ramalho do Rêgo wrote the manuscript.

\section{REFERENCES}

BALDISSERA JNC, VALENTINI G, CIAN MM, ALMEIDA CB, GUIDOLIN AF AND COIMBRA JLM. 2012. Combining ability and reciprocal effect on agronomical traits of bean. Semina: Ciências Agrárias 33: 471-480.

BARROSO PA, PESSOA AMS, MEDEIROS GDA, SILVA NETO JJ, REGO ER AND RÊGO MM. 2015. Genetic Control of Seed Germination and Physiological Quality in Ornamental Pepper. Acta Hortic 1087: 409-414.

BARROSO PA, RÊGO ER, RÊGO MM, NASCIMENTO KS, NASCIMENTO NFF, NASCIMENTO MF, SOARES WS, FERREIRA KTC AND OTONI WC. 2012. Analysis of segregating generation for components of seedling and plant height of pepper (Capsicum annuum L.) for medicinal and ornamental purposes. Acta Hortic 953: 269-276.

BARROSO PA, RÊGO ER, RÊGO MM, NASCIMENTO NFF, LEITE OS AND FERREIRA KTC. 2011. Análise de 
geração segregante para componentes de porte de planta em pimenteiras ornamentais. Hortic Bras 29: 2975-2980.

BARROSO PA, RÊGO MM, RÊGO ER AND FERREIRA KTC. 2017. Genetic effects of in vitro germination and plantlet development in chilli pepper. Genet Mol Res 16: $1-10$.

BENIN G, SILVA GO, PAGLIOSAES, LEMES C, SIGNORINI A, BECHE E AND CAPELIN MA. 2009. Capacidade de combinação em genótipos de trigo estimada por meio de análise multivariada. Pesq Agropec Bras 44: 1145-1151.

COSTA MPSD, RÊGO MM, SILVA APG, RÊGO ER AND BARROSO PA. 2016. Characterization and genetic diversity of pepper (Capsicum spp.) parents and interspecific hybrids. Genet Mol Res 15: 1-12.

CRUZ CD. 2006. Programa genes (versão Windows): aplicativo computacional em genética e estatística. Editora UFV (Universidade Federal de Viçosa), Viçosa, 382 p.

DO RÊGO, ER, NASCIMENTO MF, DO NASCIMENTO NFF, DOS SANTOS RMC, FORTUNATO FLG AND DO RÊGO MM. 2012. Testing methods for producing selfpollinated fruits in ornamental peppers. Hortic Bras 30: 669-672.

FERREIRA KTC, RÊGO ER, RÊGO MM, FORTUNATO FLG, NASCIMENTO NFF AND LIMA JAM. 2015. Combining Ability for Morpho-Agronomic Traits in Ornamental Pepper Acta Hortic 1087: 187-194.

GARDNER EJ AND EBERHART SA. 1966. A analysis and interpretetion of the variety cross diallel and related populations. Biometrics 22: 439-452.

GONÇALVES JGR, CHIORATO AF, SILVA DA, ESTEVES JAF, BOSETTI F AND CARBONELL SAM. 2015. Análise da capacidade combinatória em feijoeiro comum submetido ao déficit hídrico. Bragantia 74: 149-155.

GRIFFING B. 1956. Concept of general and specific combining ability in relation to diallel crossing systems. Aust J Biol Sci 9: 463-493.

IPGRI. 1995. Descriptores para Capsicum (Capsicum spp.). Roma: IPGRI, p. 1-51.

LEDO CAS, FERREIRA DF AND RAMALHO MAP. 2003. Análise de variância multivariada para os cruzamentos dialélicos. Ciênc Agrotec 27: 1214-1221.

MEDEIROS AM, RODRIGUES R, GONÇALVES LSA, SUDRÉ CP, OLIVEIRA HS AND SANTOS MH. 2014. Gene effect and heterosis in Capsicum baccatum var. pendulum. Ciênc Rural 44: 1031-1036.

MEDEIROS GDA, REGO ER, BARROSO PA, PESSOA AMS, REGO MM AND CRISPIM JG. 2015. Heritability of Traits Related to Germination and Morphogenesis. Acta Hortic 1087: 403-408.

MENDES UC, MIRANDA FILHO JB, OLIVEIRA AS AND REIS EF. 2015. Heterosis and combining ability in crosses between two groups of open-pollinated maize populations. Cbab 15: 235-243.
MONGKOLPORN O AND TAYLOR PWJ. 2011. Capsicum. In: Kole C. Wild crop relatives: genomic and breeding resources, Vegetables. Springer, Berlin 10: 43-57.

NASCIMENTO MF, NASCIMENTO NFF, RÊGO ER, SAPUCAY MJLC, FORTUNATO FLG AND RÊGO MM. 2011. Heterose em cruzamentos dialélicos para qualidade de frutos em pimenteiras ornamentais. Hortic Bras 29: 2948-2955.

NASCIMENTO NFF, NASCIMENTO MF, RÊGO ER, LIMA JAM, RÊGO MM, FINGER FL AND BRUCKNER CH. 2015. Intraspecific Cross-Compatibility in Ornamental Pepper. Acta Hortic 1087: 339-344.

NASCIMENTO NFF, NASCIMENTO MF, RÊGO ER, RÊGO MM, SANTOS RMC, BRUCKNER CH AND FINGER FL. 2013. Flower color variability in double and three-way hybrids of ornamental pepper. Acta Hortic 1000: 457-464.

NASCIMENTO NFF, RÊGO ER, NASCIMENTO MF, BRUCKNER CH, FINGER FL AND RÊGO MM. 2014. Combining ability for yield and fruit quality in the pepper Capsicum annuum. Genet Mol Res 13: 3237-3249.

NASCIMENTO NFF, RÊGO ER, RÊGO MM, NASCIMENTO MF AND ALVES LI. 2012. Compatibilidade em cruzamentos intra e interespecíficos em pimenteiras ornamentais. Ornam Hortic 18: 58-61.

OLIVEIRA EJ. 2014. Capacidade de combinação e heterose em maracujazeiro amarelo. Biosci J 30: 757-767.

PESSOA AMS, BARROSO PA, RÊGO ER, MEDEIROS GDA, BRUNO RLA AND RÊGO MM. 2015. Genetic divergence of physiological-quality traits of seeds in a population of peppers. Genet Mol Res 14: 12479-12488.

PESSOA AMS, RÊGO ER, CARVALHO MG, SANTOS CAP AND RÊGO MM. 2018. Genetic diversity among accessions of Capsicum annuum L. through morphoagronomic characters. Genet Mol Res 17: 1-15.

RÊGO ER AND RÊGO MM. 2016. Genetics and Breeding of Chili Pepper Capsicum spp. In: Rêgo ER, Rêgo MM and Finger FL. Production and Breeding of Chilli Peppers (Capsicum spp.). Springer 1: 1-134.

RÊGO ER AND RÊGO MM. 2018. "Ornamental Pepper", in Ornamental Crops. Ed. Van Huylenbroeck J. (Springer International Publishing Switzerland) 11: 529-565.

RÊGO ER, RÊGO MM AND FINGER FL. 2015. Methodological Basis and Advances for Ornamental Pepper Breeding Program in Brazil. Acta Hortic 1087: 309-314.

RÊGO ER, RÊGO MM, FINGER FL, CRUZ CD AND CASALI VWD. 2009. A diallel study of yield components and fruit quality in chilli pepper (Capsicum baccatum). Euphytica 168: 275-287.

RÊGO ER, RÊGO MM, FINGER FL, NASCIMENTO NFF, NASCIMENTO MF AND SANTOS RMC. 2013. Phenotypic variability and importance of characters in a 
$\mathrm{F}_{2}$ segregating generation of ornamental chili (Capsicum annuum). Acta Hortic 1000: 493-498.

RÊGO ER, RÊGO MM, MATOS IWF AND BARBOSA LA. 2011. Morphological and chemical characterization of fruits of Capsicum spp. accessions. Hortic Bras 29: 364371.

RÊGO ER, SILVA DF, RÊGO MM, SANTOS RMC, SAPUCAY MJLC AND SILVA DR. 2010. Diversidade entre linhagens e importância de caracteres relacionados à longevidade em vaso de linhagens de pimenteiras ornamentais. Ornam Hortic 16: 165-168.

ROCHA F, STINGHEN JC, GEMELI MS, COIMBRA JLM AND GUIDOLIN AF. 2014. Análise dialélica como ferramenta na seleção de genitores em feijão. Rev Ciênc Agron 45: 74-81.

SANTOS RMC, NASCIMENTO NFF, BORÉM A, RÊGO ER, RÊGO MM, FINGER FL, COCA GC, NASCIMENTO MF AND LEMOS RC. 2013. Ornamental pepper breeding: could a chili be a flower ornamental plant? Acta Hortic 1000: 451-456.

SEGATTO FB, FINGER FL, BARBOSA JG, RÊGO ER AND PINTO CMF. 2013. Effects of ethylene on the postproduction of potted ornamental peppers (Capsicum annuum). Acta Hortic 1000: 217-222.
SILVA NETO JJ, RÊGO ER, NASCIMENTO MF, SILVA FILHO VAL, ALMEIDA NETO JX AND RÊGO MM. 2014. Variabilidade em população base de pimenteiras ornamentais (Capsicum annuum L.). Revista Ceres 61: 84-89.

SPRAGUE GF AND TATUM LA. 1942. General vs. specific combining ability in single crosses of corn. J Amer Soc Agron 34: 923-932.

STOMMEL JR. 2008. Inheritance of fruit, foliar, and plant habit attributes in Capsicum. J Amer Soc Hort Sci 133: 396-407.

STOMMEL JR AND BOSLAND PW. 2006. Ornamental pepper, Capsicum annuum. In: Anderson N (Ed), Flower Breeding and Genetics: Issues, Challenges and 64 opportunities for the 21 st Century. Dordrecht, The Netherlands: Springer 1: 561-599.

\section{SUPPLEMENTARY MATERIAL}

Table SII - Seedling and plant quantitative traits for seven accessions and 42 hybrids of Capsicum annuum L. Values are means. 